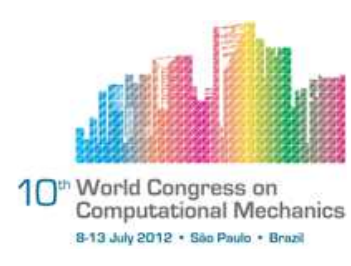

\title{
NUMERICAL SIMULATION OF TWO-PHASE FLOWS USING A HYBRID VOLUME OF FLUID AND IMMERSED BOUNDARY METHOD
}

\author{
M. M. Villar ${ }^{1}$, M. R. Pivello ${ }^{1}$, A., Sene, R. ${ }^{1}$, Silveira-Neto $^{1}$, A. M. Roma ${ }^{2}$ \\ ${ }^{1}$ Department of Mechanical Engineering, Federal University of Uberlândia, Uberlândia MG. \\ (mmvillar@mecanica.ufu.br) \\ ${ }^{2}$ Institute of Mathematics and Statistics, University of São Paulo.
}

\begin{abstract}
In this paper a simulation model is presented for the Direct Numerical simulation (DNS) of multiphase flow. This method combines the Volume of fluid model and Immersed Boundary method in order to investigate water-oil flow-pattern. The simulations were carried out on structured cartesian adaptive mesh refinement (SAMR), where the the Immersed Boundary represents the circular tube via Direct Forcing Method(DFM). The Volume of Fluid based on piecewise linear reconstruction interface, allow to define the fluid-fluid interface using a three dimensional version of the continuum surface force (CSF) model of Brackbill et al. (1992). The Navier-Stokes equations are discretized in the entire domain using a finite difference scheme, where a temporal discretization is based on SBDF scheme, with adaptive time stepping. The capabilities of the hybrid VOF-IB model are demonstrated with examples in wich complex topological changes in the interface are encountered. The overall methodology has been through a careful, series of verification tests whose results are reported. An oil-water flow-pattern application is presented.
\end{abstract}

Keywords: Two-Phase Flow, Structured Adaptive Mesh Refinement, Volume of Fluid, Immersed Boundary method.

\section{INTRODUCTION}

Multiphase flows are the source of numerous nonlinear processes of both scientific and technological relevance. These flows are characterized by a complex motion of fluid interfaces separating masses of fluids with different material properties which can undergo significant deformations and topological transitions.

Due to the multi-component nature of the flow, fluid interfaces are subjected to surface tension and this interfacial force plays a fundamental role in nearly all multiphase flows of physical interest. Moreover, multiphase flows are typically multi-scale. The important phenomena of drop coalescence and break-up as well as the generation of short capillary waves are just a few examples that exhibit the presence of multiple length scales.

Numerical methods for computing multiphase flows can be broadly divided into two types: capturing and tracking methodologies. In capturing methods, such as the continuum surface force (CSF) model [6], the level set approach [8], the phase field method [4], and the volume-of-fluid (VOF) method $[15,14,19,18]$, the fluid interface is implicitly defined 
through a globally specified scalar function (the mass density, a signed distance function, or a volume fraction) which acts as a fluid indicator. These methods capture the interface motion on an Eulerian grid and handle automatically changes in interfacial topology. Front-tracking methods [7], on the other hand, use a separate grid to explicitly follow the interface motion.

In recent years, much attention has been paid to the development of hybrid approaches [3, 19]. Combined methods also has achieved space in the scientific literature [10]. Following this philosophy, the method presented here combines the Volume of Fluid (VOF) method, to represent the fluid-fluid interface, and the Immersed Boundary method (IB), to represent the solid-fluid interface. In the present work, such combination is employed to perform the computational simulations of flow through a long pipe: the immersed boundary is usedo to model the pipe while the VOF method defines the gas-fluid interface.

Here, VOF is based on a piecewise linear interface representation (PLIC/VOF). VOF method was originally proposed by Hirt and Nichols [30] and over time has frequently received many improvements $[20,16]$. In the present work, the model is based on Young's VOF method for which the best performance in standard advection was obtained, similar as reported by Rudman [23]. The IB method embeds a direct forcing method as proposed by Uhlmann [29] and Wang [31]. Differently from [10], the collision model, to account for dissipative particle-particle and particle-wall collision, has been not considered yet. Both methods are implemented in the context structured adaptive mesh refinement in order to allow variable spatial resolution along the interface, which can be a solid-fluid interface or a fluid-fluid interface. The numerical method also evolves accordingly to a second order, semi-implicit time discretization (Semi-Backward Difference Formula - SBDF) and a second-order finite difference scheme for the spatial discretization. A multigrid-multilevel is applied to solve the pressure Poisson equation.

The main goal is to show the capability of the combined method to simulate an oilwater flow-pattern on a pipeline system, considering a structured cartesian mesh. With this goal in mind, the current work presents the governing equations and the numerical method. Section 3 is devoted to the verification of the method where three dimensional test cases are examined. In Section 4, an example of oil-water flow-pattern is presented and discussed and, finally, in Section 5 the conclusions are presented.

\section{GOVERNING EQUATIONS AND NUMERICAL SCHEME}

The combined model presented here consists of two main parts: one considers the presence of deformable interfaces (Volume of Fluid model) and the other one considers the presence of solid interfaces (Immersed Boundary Method). Both of them are embedded on an incompressible, variable-density flow governed by the Navier-Stokes equations.

\subsection{Governing equations}

The incompressible, variable-density, Navier-Stokes equations with surface tension can be written as

$$
\begin{aligned}
\rho\left[\mathbf{u}_{t}+(\mathbf{u} \cdot \nabla) \mathbf{u}\right] & =\nabla \cdot\left[\mu\left(\nabla \mathbf{u}+\nabla \mathbf{u}^{\dagger}\right)\right]-\nabla p+\rho \mathbf{g}+\mathbf{f}_{\sigma}+\mathbf{f}_{s}, \\
\nabla \cdot \mathbf{u} & =0
\end{aligned}
$$

where $\mathbf{u}=(u, v, w)$ is the fluid velocity, $\rho \equiv \rho(\mathbf{x}, t)$ is the fluid density, and $\mu \equiv \mu(\mathbf{x}, t)$ is the dynamic viscosity. Here, $p$, $\mathbf{g}$, and $\mathbf{f}_{\sigma}$ are the pressure, the gravitational acceleration, and the 
surface tension force, respectively. The source term $\mathbf{f}_{s}$ accounts for the fluid-solid coupling (physical units $\mathrm{N} / \mathrm{m}^{3}$ ).

For two-phase flows the volume fraction $C(\mathbf{x}, t)$ is introduced, and through it is possible to define the density and viscosity as

$$
\begin{aligned}
& \rho(C)=C \rho_{1}+(1-C) \rho_{2}, \\
& \mu(C)=C \mu_{1}+(1-C) \mu_{2},
\end{aligned}
$$

with $\rho_{1}, \rho_{2}$ and $\mu_{1}, \mu_{2}$ the density and viscosity of the first and second fluids respectively.

The interface is advected with the local fluid velocity, using the following advection equation to the volume fraction:

$$
\partial_{t} C+\nabla \cdot(C \mathbf{u})=0
$$

\subsection{Discretization in time}

The time discretization scheme presented here is similar to the scheme presented in [7] with minor modifications, which allow different semi-implicit schemes to be chosen from accordingly to the necessity. The second-order semi-implicit scheme is given by

$$
\begin{aligned}
& \frac{\rho^{n+1}}{\Delta t}\left(\alpha_{2} \mathbf{u}^{n+1}+\alpha_{1} \mathbf{u}^{n}+\alpha_{0} \mathbf{u}^{n-1}\right)=\beta_{1} f\left(\mathbf{u}^{n}\right)+\beta_{0} f\left(\mathbf{u}^{n-1}\right)+ \\
& \lambda\left[\theta_{2} \nabla^{2} \mathbf{u}^{n+1}+\theta_{1} \nabla^{2} \mathbf{u}^{n}+\theta_{0} \nabla^{2} \mathbf{u}^{n-1}\right]-\nabla p^{n}+\rho^{n+1} \mathbf{g}, \\
& \nabla \cdot \mathbf{u}^{n+1}=0,
\end{aligned}
$$

where $\lambda=|\mu|_{\infty}$ and $f(u)$ the forcing term

$$
f(\mathbf{u})=-\lambda \nabla^{2} \mathbf{u}+\nabla \cdot\left[\mu\left(\nabla \mathbf{u}+\nabla \mathbf{u}^{T}\right]-\mathbf{u} \cdot \nabla \mathbf{u}+\mathbf{f}_{\sigma} .\right.
$$

The parameters $\alpha_{i}, \beta_{i}$, and $\theta_{i}$ are

$$
\begin{aligned}
\alpha_{0} & =\frac{(2 \gamma-1) \omega^{2}}{1+\omega}, \\
\alpha_{1} & =(1-2 \gamma) \omega-1, \\
\alpha_{2} & =\frac{1+2 \gamma \omega}{1+\omega}, \\
\beta_{1} & =-\gamma \omega, \\
\beta_{0} & =1+\gamma, \\
\theta_{0} & =\frac{c}{2}, \\
\theta_{1} & =1-\gamma-\left(1+\frac{1}{\omega}\right) \frac{c}{2}, \\
\theta_{2} & =\gamma+\frac{c}{2 \omega},
\end{aligned}
$$

where $\omega=\Delta t_{n+1} / \Delta t_{n}$ is the ratio between two consecutive time steps. That family of numerical schemes involving the two parameters $\gamma$ and $c$, as showed in [1], includes

- Cranck-Nicolson Adams-Bashforth (CNAB): $(\gamma, c)=(0.5,0.0)$. 
- Modified Crank-Nicolson Adams-Bashforth (MCNAB): $(\gamma, c)=(0.5,0.125)$.

- Crank-Nicolson Leap Frog (CNLF): $(\gamma, c)=(0.0,1.0)$.

- Semi-Backward Difference (SBDF): $(\gamma, c)=(1.0,0.0)$.

In the present work, the above SBDF temporal time discretization is chosen in all cases, with a variable size of time step.

To handle the pressure-velocity coupling, given by (5) and (6), the fractional time-step method is proposed

$$
\begin{aligned}
& \frac{\rho^{n+1}}{\Delta t}\left(\alpha_{2} \mathbf{u}^{*}+\alpha_{1} \mathbf{u}^{n}+\alpha_{0} \mathbf{u}^{n-1}\right)=\beta_{1} f\left(\mathbf{u}^{n}\right)+\beta_{0} f\left(\mathbf{u}^{n-1}\right)+ \\
& \lambda\left[\theta_{2} \nabla^{2} \mathbf{u}^{*}+\theta_{1} \nabla^{2} \mathbf{u}^{n}+\theta_{0} \nabla^{2} \mathbf{u}^{n-1}\right]-\nabla p^{n}+\rho^{n+1} \mathbf{g}, \\
& \mathbf{u}^{n+1}=\mathbf{u}^{*}-\frac{\Delta t}{\alpha_{2}} \frac{\nabla q}{\rho^{n+1}} \\
& \nabla \cdot \mathbf{u}^{n+1}=0 .
\end{aligned}
$$

Once the provisional velocity $\mathbf{u}^{*}$ is computed from (9) imposing $\mathbf{u}^{*} \doteq \mathbf{u}^{n+1}$ on the boundaries, it is projected onto the space of divergence-free vector fields. This is accomplished by solving the Poisson equation for $q$ defined by (10) and (11) along with homogeneous Neumann boundary conditions $\partial q / \partial \mathbf{n}=0$, where $\mathbf{n}$ is the exterior normal to the domain.

Multilevel-multigrid methods are employed to solve for both the provisional vector field, $\mathbf{u}^{*}$ in (9), and the pressure increment $q$. The last one is obtained from

$$
\nabla \cdot\left[\frac{1}{\rho^{n+1}} \nabla q\right]=\frac{\alpha_{2}}{\Delta t} \nabla \mathbf{u}^{*}
$$

\subsection{Discretization in space}

Space is discretized using the structured adaptive mesh refinement, which is based on the hierarchical grid structure proposed by Berger and Colella [5] and on the adaptive IB method introduced by Roma et al. [22]. In this scheme, regions of the flow bearing special interest are covered by block-structured grids, defined as a hierarchical sequence of nested, progressively finer levels (composite grids). Each level is formed by a set of disjoint rectangular grids and the refinement ratio between two successive refinement levels are constant and equal to two. Ghost cells are employed around each grid, for all the levels, and underneath fine grid patches to formally prevent the finite difference operators from being redefined at grid borders and at interior regions which are covered by finer levels. Values defined in these cells are obtained from interpolation schemes, usually with second or third order accuracy, and not from solving the equations of the problem. A staggered composite grid is used. The discretizations of the Laplacian, gradient and divergence differential operators are performed by standard, cell-centered second order stencils.

\subsection{Volume of Fluid Method}

To solve the volume fraction advection equation (Eq. 4), a piecewise-linear geometrical Volume of Fluid based on [24] scheme is used. Geometrical VOF schemes classically proceed in two steps: 


\section{Interface reconstruction.}

2. Geometrical flux computation and interface advection.

Interface reconstruction is preformed using a piecewise-planar interface representation in each cell defined by the equation

$$
\mathbf{m} \cdot \mathbf{x}=\alpha .
$$

Given the interface normal $\mathbf{m}$ and the volume fraction $\mathrm{C}$ in a given cell, $\alpha$ can be determined uniquely using analytical relations ([15], [16], [25]). The interface normal $\mathbf{m}$ can be approximated by considering the volume fractions in a neighborhood of the cell considered, where the Mixed-Youngs-Centred (MYC) implementation of [2] is used. Once interface reconstruction has been performed, direction-split geometrical fluxes can be computed easily on regular Cartesian grids.

As shown by [15], the resulting advection scheme preserves a sharp interface and has been shown to be close to second-order accurate for practical applications. While this scheme is not strictly conservative [20], errors in mass conservation for intricate problems are usually less than $0.01 \%$.

\subsection{Surface tension}

The surface tension term $\left(\mathbf{f}_{\sigma}=\sigma \kappa \delta_{s} \mathbf{n}\right)$, in the momentum equation (1), is aproximated based on the original Continuum-Surface-Force (CSF) approach of [6]. To reduce the parasitic currents which are always present in this approach, the Height-Function technique is used for an accurate curvature evaluation.

Recently, [19] showed that the combination of a balanced-force surface tension discretization and a Height-Function curvature estimation is sufficient to solve the problem of parasitic currents. However, the Heigh-Function technique ([9], [11]) presents inconsistent approximations when the radius of curvature of interface becomes comparable to mesh size. In this case, other techniques must be coupled with HF to supply a better approximation. In the present work, for the inconsistent points Shirani's discretization is used [26].

\subsection{Immersed Boundary Method}

The momentum source term $\mathbf{f}_{s}$ accounts for the presence of the rigid body. The source term $\mathbf{f}_{s}$ is evaluated by direct forcing methodology, proposed by [29]. The main characteristic of this method is that it is not necessary to use ad-hoc constants and it allows for the non-slip condition modeling along the immersed interface.

The source term $\mathbf{f}_{s}$ is defined in all domain $\Omega$, but it presents values different from zero only at the points that coincide with the immersed geometry,

$$
\mathbf{f}_{s}(\mathbf{x}, t)=\left\{\begin{array}{rll}
\mathbf{F}_{s}(\mathbf{X}, t) & \text { se } & \mathbf{x}=\mathbf{X} \\
0 & \text { se } & \mathbf{x} \neq \mathbf{X}
\end{array}\right.
$$

where $\mathbf{x}$ is the position of any particle in the fluid and $\mathbf{X}$ is the position of any point on the rigid interface, and the Lagrangian force $\mathbf{F}_{s}(\mathbf{X}, t)$ is

$$
\begin{aligned}
\mathbf{F}_{s}(\mathbf{X}, t) & =\rho(\mathbf{X}, t)\left[\mathbf{U}_{t}+(\mathbf{U} \cdot \nabla) \mathbf{U}\right]-\nabla \cdot\left[\mu(\mathbf{X}, t)\left(\nabla \mathbf{U}+\nabla \mathbf{U}^{\dagger}\right)\right] \\
& +\nabla P-\rho(\mathbf{X}, t) \mathbf{g}-\mathbf{F}_{\sigma},
\end{aligned}
$$


where $\mathrm{U}$ and $P$ belong to the Lagragian domain and can be given by interpolation of velocities and pressure, respectively, from Eulerian points near the immersed body.

Force (15) may be rewritten as

$$
\mathbf{F}_{s}(\mathbf{X}, t)=\rho(\mathbf{X}, t) \frac{\alpha_{2} \mathbf{U}^{n+1}-\alpha_{2} \mathbf{U}^{*}+\alpha_{2} \mathbf{U}^{*}+\alpha_{1} \mathbf{U}^{n}+\alpha_{0} \mathbf{U}^{n-1}}{\Delta t}+\mathbf{R} \mathbf{H} \mathbf{S}^{n, n-1},
$$

where $\mathbf{U}^{*}$ is a temporary parameter, $\Delta t$ is the time step and RHS is the right had side composed by advective term , diffusive term, gradient pressure term, gravitational term, and interfacial term force. Note that (16) is solved by equations (17) and (18) at the same time step:

$$
\begin{aligned}
& \rho(\mathbf{X}, t) \frac{\alpha_{2} \mathbf{U}^{*}+\alpha_{1} \mathbf{U}^{n}+\alpha_{0} \mathbf{U}^{n-1}}{\Delta t}+\mathbf{R H} \mathbf{S}^{n, n-1}=0, \\
& \mathbf{F}_{s}(\mathbf{X}, t)=\rho(\mathbf{X}, t) \frac{\alpha_{2} \mathbf{U}^{n+1}-\alpha_{2} \mathbf{U}^{*}}{\Delta t},
\end{aligned}
$$

where $\mathbf{U}^{n+1}=U_{F I}$ is the immersed boundary, prescribed velocity.

Equation (17) is solved on the Eulerian domain, i.e. the solution of equation (1) with $\mathbf{f}_{s}=0$. The temporary velocity $\mathbf{u}^{*}$ is interpolated to the Lagrangean domain and becomes $\mathbf{U}^{*}$ and is used on equation (18). After that, $\mathbf{F}_{s}(\mathbf{X}, t)$ is smeared out to the Eulerian mesh using a distribution function $D$

$$
\begin{gathered}
\mathbf{f}_{s}(\mathbf{x})=\sum_{\Gamma} D_{h}(\mathbf{x}-\mathbf{X}) \mathbf{F}_{s}(\mathbf{X}) \Delta V, \\
D_{h}(\mathbf{x}-\mathbf{X})=\frac{1}{h^{3}} W_{g}\left(\frac{x-X}{h}\right) W_{g}\left(\frac{y-Y}{h}\right) W_{g}\left(\frac{z-Z}{h}\right), \\
W_{g}(r)=\left\{\begin{array}{r}
1-|r| \text { se } 0 \leq|r| \leq 1, \\
0 \quad \text { se } 1<|r| .
\end{array}\right.
\end{gathered}
$$

where $r=\frac{x-X}{h}, h$ is the Eulerian mesh spacing, and $\Delta V$ is the influence range volume (see [29]).

Finally, the Eulerian velocity is updated by equation (22)

$$
\mathbf{u}^{* *}=\mathbf{u}^{*}+\frac{\Delta t}{\rho(\mathbf{x}, t) \alpha_{2}} \mathbf{f}_{s} .
$$

Equations (18)-(22) can be iterated few fixed times or until some tolerance error has been attained. It is important to note that, in a multiphase flow, the density ratio can reach high values. We have noticed that when running IB Method in the context of multiphase flows, especially, when the density ratio is higher than 10 , the error between $\rho(\mathbf{X}, t)$ and $\rho(\mathbf{x}, t)$ can impact the multi-direct force convergence. That is the reason why in those cases, in equations (18)-(22), the density is neglected. Note that this does not affect the final result since when substituting (18) into (22) the densities cancel out.

\section{VERIFICATION AND VALIDATION}

Verification/validation is an important step in the development of any numerical tool. In the present work, the we use the method of manufactured solutions (MMS) to verify the convergence rate of the Eulerian set of equations only, while validation tests are applied to the other numerical techniques as curvature and interfacial force computations, and IB Method implementation. 


\subsection{Verification of an incompressible solution}

MMS consists in obtaining a priori a known analytical solution for the system of governing equations. Those manufactured solutions require the addition of a source term to the original differential equation coming from a set of chosen function which will play the role of the exact solution. Here, we choose

$$
\begin{aligned}
& p_{e}=\cos \left(\alpha_{s} \pi x+\beta_{s} \pi y+\gamma_{s} \pi z+\delta_{s} t\right)^{2}, \\
& u_{e}=\sin \left(\alpha_{s} \pi x+\beta_{s} \pi y+\gamma_{s} \pi z+\delta_{s} t\right)^{2}, \\
& v_{e}=-\cos \left(\alpha_{s} \pi x+\beta_{s} \pi y+\gamma_{s} \pi z+\delta_{s} t\right)^{2}, \\
& w_{e}=\frac{\alpha_{s}}{\gamma_{s}} \cos \left(\alpha_{s} \pi x+\beta_{s} \pi y+\gamma_{s} \pi z+\delta_{s} t\right)^{2}+\frac{\beta_{s}}{\gamma_{s}} \cos \left(\alpha_{s} \pi x+\beta_{s} \pi y+\gamma_{s} \pi z+\delta_{s} t\right)^{2}, \\
& \rho_{e}=1+0.1 \sin \left(\alpha_{s} \pi x+\beta_{s} \pi y+\gamma_{s} \pi z+\delta_{s} t\right)^{2}, \\
& \mu_{e}=1+0.2 \cos \left(\alpha_{s} \pi x+\beta_{s} \pi y+\gamma_{s} \pi z+\delta_{s} t\right)^{2},
\end{aligned}
$$

where the subscript $e$ stands for "exact". Observe that the divergence of (24)-(26) is zero, as it should. Functions (23)-(24) are written as functions of constant parameters $\alpha_{s}, \beta_{s}, \gamma_{s}$, and $\delta_{s}$. Such parameters may be chosen to apply MMS for a wider range of numerical cases. For example, if $\delta_{s}$ is set to zero, only the spatial derivatives influence the convergence rate of the scheme. On the other hand, if $\alpha_{s}, \beta_{s}$ and $\gamma_{s}$ are set to zero, only temporal scheme is tested.

The computational domain considered is the cube $[0,1] \times[0,1] \times[0,1]$, aligned with the coordinate axis. The time step is controlled by CFL condition, with a CFL constant chosen to be 0.5 . The parameters $\alpha_{s}, \beta_{s}$, and $\gamma_{s}$ are set to 2 , and $\delta_{s}=1$. The domain is discretized with a fixed composite mesh showed in Figure 1.

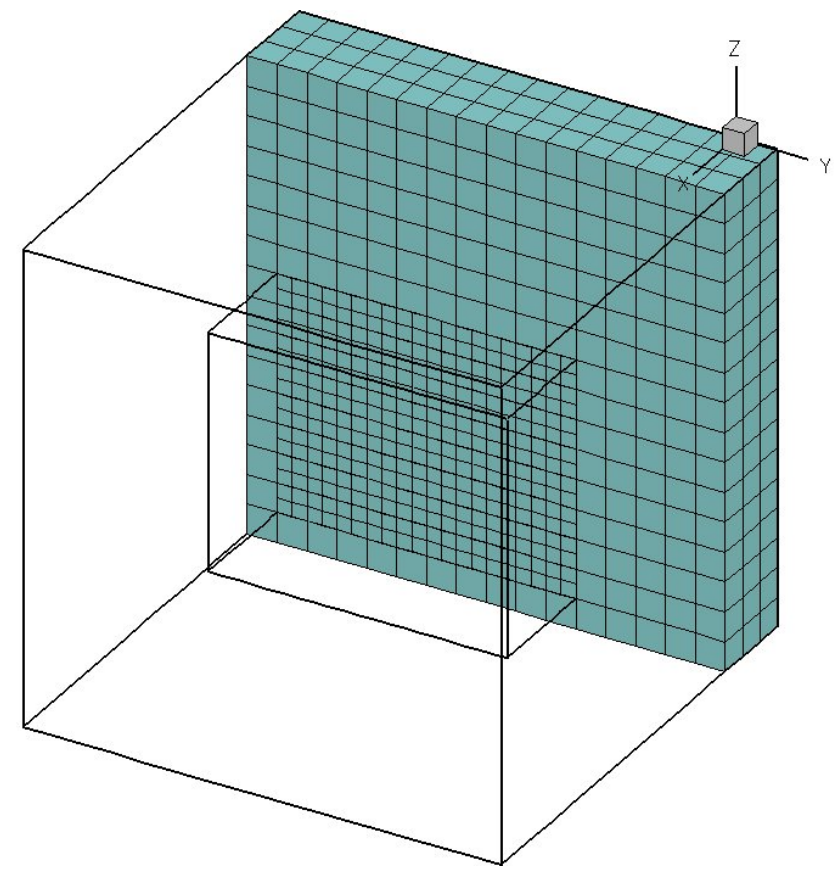

Figure 1. Composite mesh used for convergence analysis (the mesh does not change in time).

We employ the $L_{2}$-norm for the convergenc analysis:

$$
L_{2}=\frac{1}{V_{\Omega}}\left(\sum\left|\phi_{(i, j, k)}^{h}-\phi_{(i, j, k)}^{e}\right|^{2} \Delta x \Delta y \Delta z\right)^{\frac{1}{2}}
$$


where $V_{\Omega}$ is the domain volume. The error decay ratio is defined as

$$
r_{e}=\log \frac{\Phi_{2 h}}{\Phi_{h}}
$$

where $\Phi_{2 h}$ is the global error on a grid with spacing $2 h$. The convergence rate $q$ is defined as $q \approx r_{e} / \log (2)$.

Table (1) displays the grid refinement ${ }^{1}$, the decay of $L_{2}$ norm and the resulting convergence rate $q$ for Dirichlet condition obtained from the exact solution. It is worth noting that at least a convergence rate equals to two is obtained for all velocities and at least equal to first order for the pressure. The values of error and convergence rate are measured at $t=0.1 \mathrm{~s}$.

\begin{tabular}{lccccccc}
\hline \multicolumn{7}{c}{ Convergence rate test } \\
\hline & {$[16]^{3} L 1$} & $q$ & {$[32]^{3} L 1$} & $q$ & {$[64]^{3} L 1$} & $q$ & {$[128]^{3} L 1$} \\
\hline$\left\|u-u_{e}\right\|_{2}$ & $1.6927 \mathrm{e}-02$ & 1.97 & $4.3227 \mathrm{e}-03$ & 2.00 & $1.0803 \mathrm{e}-03$ & 2.01 & $2.6807 \mathrm{e}-04$ \\
$\left\|v-v_{e}\right\|_{2}$ & $1.7351 \mathrm{e}-02$ & 1.98 & $4.4058 \mathrm{e}-03$ & 2.00 & $1.0999 \mathrm{e}-03$ & 2.00 & $2.7486 \mathrm{e}-04$ \\
$\left\|w-w_{e}\right\|_{2}$ & $3.1286 \mathrm{e}-02$ & 1.97 & $8.0085 \mathrm{e}-03$ & 2.01 & $1.9851 \mathrm{e}-03$ & 2.03 & $4.8464 \mathrm{e}-04$ \\
$\left\|p-p_{e}\right\|_{2}$ & 0.6372 & 1.60 & 0.2102 & 1.69 & $6.5002 \mathrm{e}-02$ & 1.69 & $2.0070 \mathrm{e}-02$ \\
\hline
\end{tabular}

Table 1. Obtained convergence rates for Dirichlet boundary conditions on a composite mesh.

\subsection{Validation test with a stationary droplet}

A common test for the accuracy of the surface tension representation in multiphase flow methods is a static cylindrical droplet $[19,28]$. That is, the exact solution is $\mathbf{u}=\mathbf{0}$, thus the droplet should not move. However, when the surface tension is sufficiently high and there is significant, numerically introduced anisotropy in its representation, artificial nonzero velocities can be generated. These "spurious currents", while small in magnitude, could affect the accuracy of computations for large tension forces and relatively coarse grids. This numerical problem has been well documented for both tracking (see for example [28]) and volume-of-fluid methods [17].

This simple flow is characterized by the Laplace number, $L a=\sigma \rho D / \mu^{2}$, where $D$ is the diameter of the droplet, and by the ratios $\rho_{1} / \rho_{2}$, and $\mu_{1} / \mu_{2}$. Here, we fix the viscosity and density ratios to one. A dimensionless measure of the strength of the spurious currents is the magnitude of the maximum non-dimensional velocity for a given simulation, where $U_{\sigma}=\sqrt{\sigma / \rho D}$ and $t_{\sigma}=\sqrt{\rho D^{3} / \sigma}$ are the reference scales.

The computational domain is a cube $[0,2] \times[0,2] \times[0,2]$. The droplet is placed at the center of the cube with $D=0.8$. Periodic boundary conditions are adopted. The mesh size is equal $3.125 \cdot 10^{-2}$ and the divergence criterion is set to $10^{-6}$. For the time-explicit discretization of the surface-tension term, numerical stability requires that the timestep be smaller than the period of the shortest capillary wave, that is, $\Delta t \leq \sqrt{\frac{\rho \Delta^{3}}{\pi \sigma}}$.

Figure 2 illustrates the evolution of $L_{2}$-norm of velocity field in time for a range of Laplace numbers (as indicated in the legend). As showed by [18], the $L_{2}$-norm(u) presents an exponential decrease in time, at all Laplace numbers.

\footnotetext{
${ }^{1}$ We refer to the composite adaptive meshes using the notation $m \times n \times o L p$ which stands for a mesh with an $m \times n \times o$ coarsest (base) level and $p$ levels of refinement (total, from the coarsest to the finest level)
} 


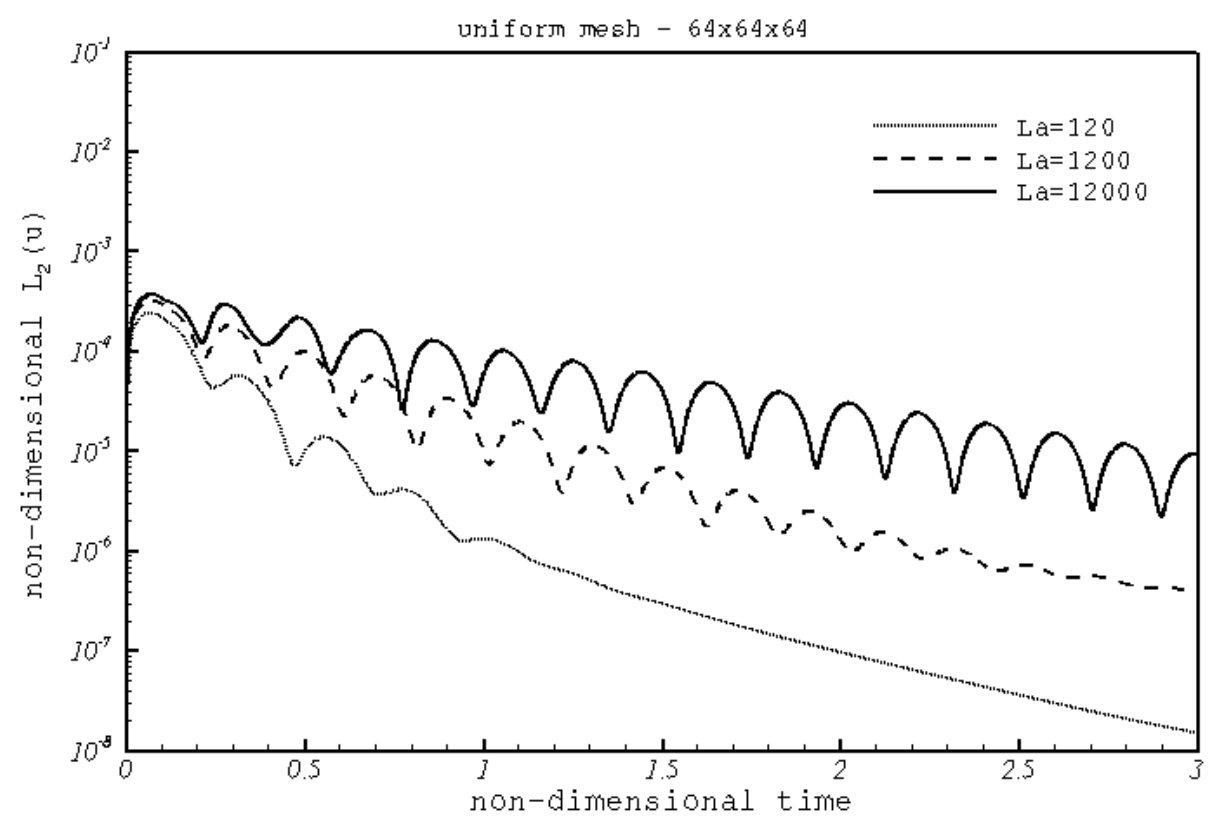

Figure 2. Evolution of spurious currents around a circular droplet.

\subsection{Validation test for the IB Method}

The Hagen-Poiseuille flow is another CFD problem used in code validation, which has an analytical solution and ensure no-slip conditions in the pipe wall. The equations governing the Hagen-Poiseuille flow can be derived directly from the NavierStokes equations in cylindrical coordinates which presents the following parabolic velocity profile:

$$
u_{x}=-\frac{1}{4 \mu} \frac{\partial p}{\partial x}\left(R^{2}-r^{2}\right),
$$

where $R$ is the pipe radius, $x$ and $r$ are the cylindrical coordinates in horizontal and radial directions, respectively.

In the present work, an unstructured mesh is used to represent the pipe and the IB Method models the no-slip condition on the pipe wall. The same procedure of MMS is again applied but the source term is replaced by a constant, which play the role of a pressure difference $(\partial p / \partial x)$. The source term is applied only at the cells located inside the pipe (for those, $\partial p / \partial x=1)$. In the complementary domain no pressure gradient is imposed.

The simulation domain is $\Omega=[0,1] \times[0,0.5] \times[0,0.5]$, the pipe length is 1 , and radius $R=0.2$. The accuracy analysis is taken at $t=10 \mathrm{~s}$, considering both composite and uniform meshes. The time step is given by a $\mathrm{CFL}=0.6$, for Reynolds $R e=100$, with $\rho=0.1$ and $\mu=0.002$. The boundary conditions adopted are periodic in $x$ direction, homogeneous Neumann in $y$ and $z$ directions. Figure 3 shows our pipe modeled by an unstructured mesh embeded in a Cartesian mesh with local refinement.

Velocity profiles at $x=0.5, y=0.25$, and $t=10.0 \mathrm{~s}$ are shown in Figure 4, where the uniform mesh $64 \times 32 \times 32$, and two others refined mesh $64 \times 32 \times 32 L 1,64 \times 32 \times 32 L 2$ are used in comparisons with the analytical solution. Erros obtained on the several meshes in Figure 4 , and the relative error for the finest mesh is about $0.1 \%$. 


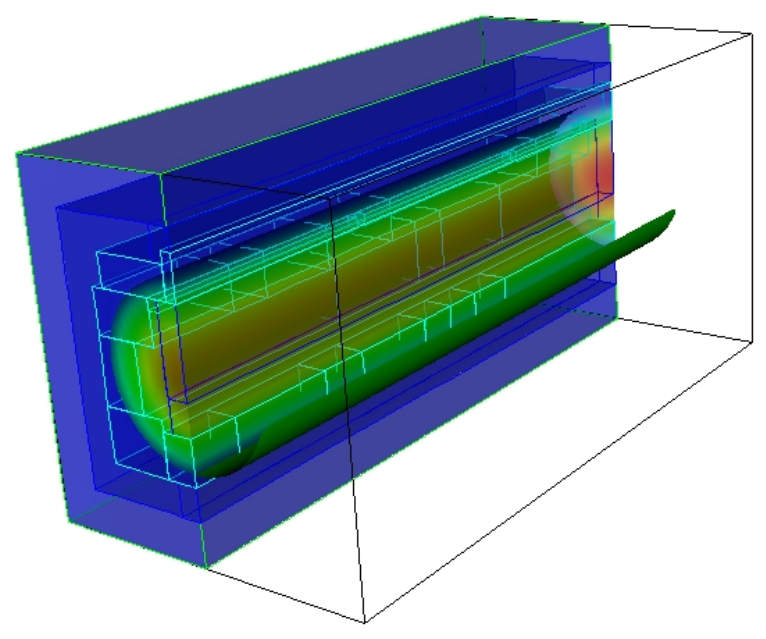

Figure 3. Pipe discretized by an unstructured mesh embeded in a Cartesian mesh with two levels of refinement.

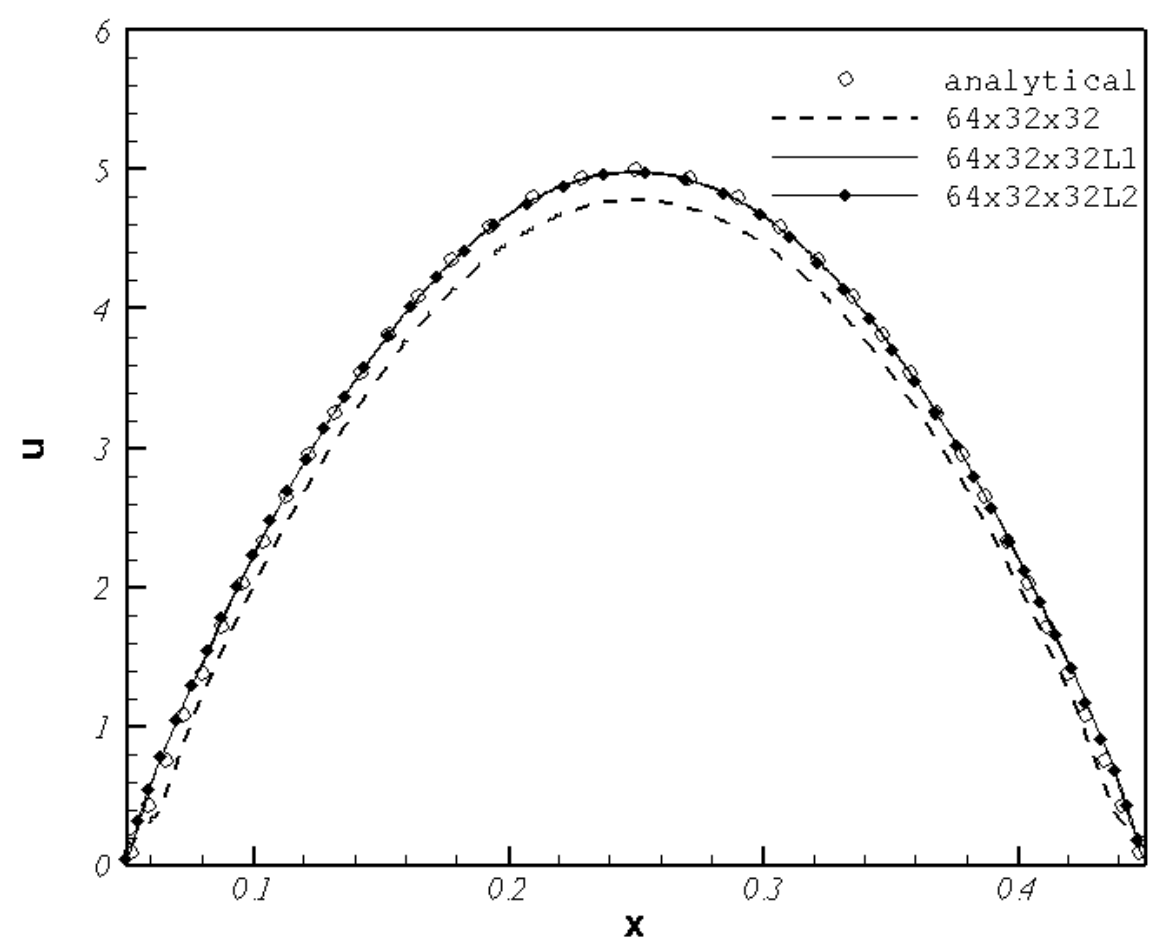

Figure 4. Velocity profile at $x=0.5, y=0.25$, and $t=10.0 \mathrm{~s}$ for the horizontal velocity in several different meshes.

\section{RESULTS}

As an application of the numerical methodology described before, an oil-water dispersion flow is chosen to illustrate the hybrid approach VOF/IB Method. Dispersed flows are 
flows in which one phase is either fully or partially dispersed into the other. In other words, when one of the phases is no longer continuou, but rather exists as droplets flowing immersed in the continuous phase, the flow is in the dispersed flow category. Depending on flow conditions, several distributions of droplets can appear. At low water cut and intermediate mixture velocity, for example, a dense layer of water droplets may appear near the pipe wall.

In liquid-liquid flow systems, it is important to understand the nature of the interactions between the phases and to observe the ways in which the phases are distributed over the cross section of the pipe. The flow pattern, usually, will not depend only on the flow behavior but also on the interfacial velocities, and on the distribution of the fraction occupied by each phase over the cross section of the pipe [27].

To simulate the flow-patterns, a large domain is required and the chosen boundary conditions can also contribute to the domain size. In recent studies, [13] and [12], recommended a pipe length of 40-80 diameters for a complete developed flow regimes without boundary influence. For 3D simulations, those kinds of long domains become impractible for our serial codes (a parallel version is underway). For a oil-water flow dispersion simulations the parameters presented in Table 2, were adopted.

\begin{tabular}{ll}
\hline & \multicolumn{1}{c}{ VOF/FI } \\
\hline Parameters & Values \\
\hline computational domain & {$[0 ; 1.024] \mathrm{m} \times[0 ; 0.064] \mathrm{m} \times[0 ; 0.064] \mathrm{m}$} \\
mesh size & $0.002 \mathrm{~m}$ \\
time step & variable \\
pipe radius & $0.054 \mathrm{~m}$ \\
oil density & $905 \mathrm{Kg} / \mathrm{m}^{3}$ \\
oil viscosity & $0.601 \mathrm{Pa.s}$ \\
water density & $995 \mathrm{Kg} / \mathrm{m}^{3}$ \\
viscosity water & $0.001 \mathrm{Pa.s}$ \\
surface tension & $0.00854 \mathrm{~N} / \mathrm{m}$ \\
\hline
\end{tabular}

Table 2. Parameter used for water-oil dispersion flow.

The simulations are performed in a adaptive mesh with 2 levels of refinement and periodic boundary condition in $x$ direction. In $y$ and $z$ directions, homogeneous Neumann boundary conditions are chosen. Oil and water are initially fully segregated. The volume fraction distribution of the liquid-liquid phases in the computational domain is initialized with a volume fraction of 0.5 and a sinusoidal disturbed free surface with a liquid level $z_{i}$ following the function:

$$
z_{i}=z_{0}+A_{i} * \sin \left(2 \pi \frac{x}{p_{i}}\right)
$$

where $z_{0}$ is the pipe center with $z_{0}=0.032, A_{i}=0.1 D$, and $p_{i}=0.5 L . D$ and $L$ are the pipe diameter and length, respectively. The initial interface disturbance is showed on Figure 5, where the iso-surface correspond to the liquid volume fraction of 0.5. The initial velocities of liquid-liquid phases are set to $u_{w}=u_{o}=2.0 \mathrm{~m} / \mathrm{s}$, which correspond to the superficial velocities $V_{s_{w}}=V_{s_{o}}=1.0 \mathrm{~m} / \mathrm{s}$. The driving pressure force in the horizontal direction is a constant with $\Delta p=9150 \mathrm{~Pa} / \mathrm{m}$.

In the transient simulation (Figure 6), the pre-set sinusoidal free surface configuration was quickly transformed into a thinner packed layer with water droplets, run up to time $1.5 \mathrm{~s}$. In this case, the interfacial mixing region grows with an increase in velocity and the two types of dispersions can coexist. Both phases retain their continuity at the top and the bottom of the 


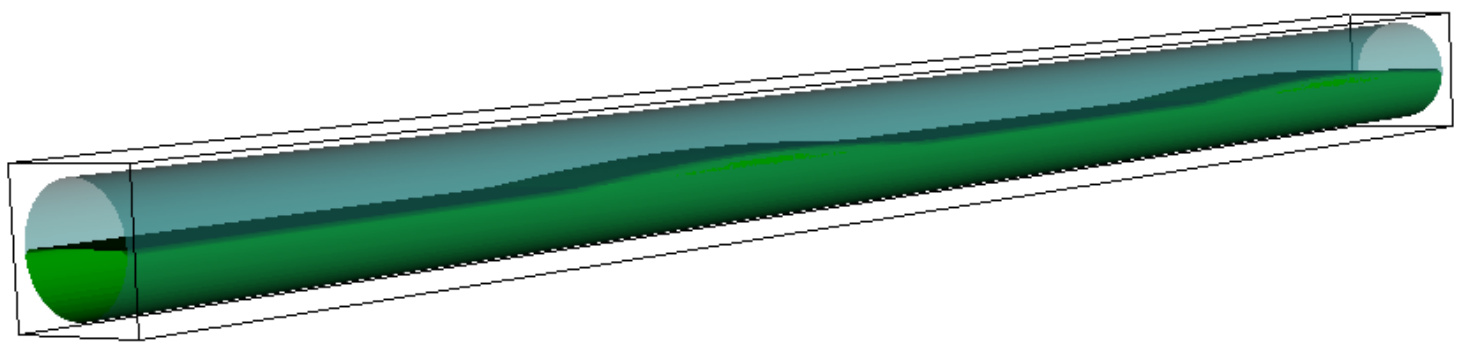

Figure 5. Isosurface of initial interface disturbance for the liquid volume fraction of 0.5 .

pipe but with each phase dispersed, at various degrees, into the continuum of each other. The flow regime is defined as dispersion of oil-in-water and water-in-oil flow (o/w \& w/o).

The dispersion of the oil-water flow in a horizontal pipe is presented in terms of flow pattern maps. Figur 7 shows the flow pattern map for a horizontal oil-water flow based on [21]. The superficial velocity, obtained when the regime, is attained corresponds to $V_{s_{w}}=2.75 \mathrm{~m} / \mathrm{s}$ and $V_{s_{o}}=1.6 \mathrm{~m} / \mathrm{s}$, which stands for Do/w \&w/o pattern.

\section{CONCLUSIONS}

A hybrid Volume-of-Fluid/IB Method for the computer simulation of transient incompressible flows given by the Navier-Stokes equations with rigid immersed bodies with prescribed velocities is implemented in an adaptive mesh refinement framework. The overall methodology is verified and validate carefully through a series of tests (including manufactured solutions and particular flow cases with known solutions). As shown by an application of the methodology to a oil-water dispersion flow problem, the methodology succeeds in predicting the main qualitatively important flow features. Future work will be concerned with the parallelization of the methodology and validating the numerical simulations with experimental data and statistical parameters such as a complete flow map pattern.

\section{Acknowledgements}

Financial support for this research was provided in part by PETROBRAS, FAPEMIG, and by CNPq (grants \# 307348/2008-3, \# 309433/2011-8 and \#151953/2008-2. ). Computer simulations were performed at MFLab, FEMEC-UFU. 


$$
\equiv
$$




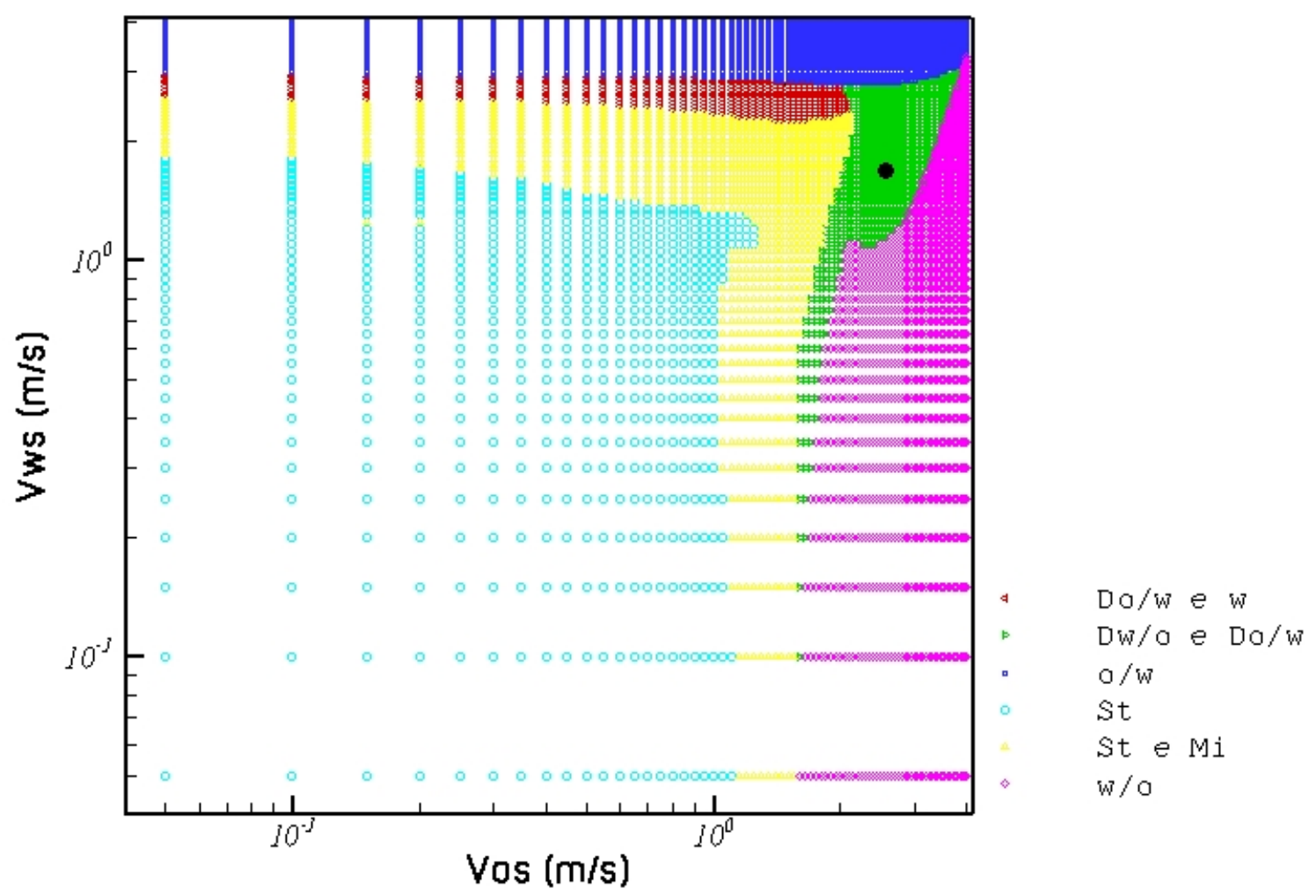

Figure 7. Map of an oil-water flow in a horizontal pipe. 


\section{References}

[1] Ascher, U. M, Ruuth, S. J. and Wetton, B. T. Implicit-explicit methods for timedependent pde's. SIAM J. Numer. Anal., 32:797-823, 1997.

[2] Aulisa, E., Manservisi, S., Scardovelli, R. and Zaleski, S. A geometrical area-preserving volume-of-fluid advection method. J. Comput. Phys., 192:355-364, 2003.

[3] Aulisa, E., Manservisi, S., Scardovelli, R. and Zaleski, S. A mixed markers and volumeof-fluid method for the reconstruction and advection of interfaces in two-phase and freeboundary flows. J. Comput. Phys., 188:611-639, 2003.

[4] Badalassi, V. E., Ceniceros, H. D. and Banerjee, S. Computation of multiphase systems with phase field models. J. Comput. Phys., 190:371-397, 2003.

[5] Berger, M. J. and Colella, P. Local adaptive mesh refinement for shock hydrodynamics. J. Comput. Phys., 82:64-84, 1989.

[6] Brackbill, J. U., Kothe, D. B. and Zemach, C. Acontinuum method for modeling surface tension. J. Computational Physics, 100:335-354, 1992.

[7] Ceniceros, H. D., Roma, A. M., Silveira-Neto, A. S. and Villar, M. M. A robust, fully adaptive hybrid level-set/front-tracking method for two-phase flows with an accurate surface tension computation. Commun. Comput. Phys., 8:51-59, 2010.

[8] Chang, Y. C., Hou, T. Y., Merriman and Osher, S. A level set formulation of eulerian interface capturing methods for incompressible fluid flows. J. Comput. Phys., 124:449464, 1996.

[9] Cummins, S. J., Francois, M. M. and Kothe, D. B. Estimating curvature from volume fractions. Computers and Structures, 83:425-434, 2005.

[10] Deen, N. G., van Sint Annaland, M. and Kuipers, J. A. M. Direct numerical simulation of complex multi-fluid flows using a combined immersed boundary and volume of fluid approach. Fith International Conference on CFD in the Process Industries, 2006.

[11] Francois, M., Cummins, E. Dendy, E., Kothe, D., Sicilian, J. and Willians, M. A balanced-force algorithm for continuous and sharp interfacial surface tension models within a volume tracking framework. J. Computational Physics, 213:141-173, 2006.

[12] Frank, T. A numerical simulation of slug flow regime for an air-water two-phase flow in horizontal pipes. The $11^{t} h$ International Topical Meeting on Nuclear Reactor ThermalHydraulics, 2005.

[13] Fukano, T. and Inatomi, T. Analysis of liquid film formation in a horizontal annular flow by dns. International Journal of Multiphase Flow, 28:1413-1430, 2003.

[14] Fuster, D., Agblagah, G., Josserand, C., Popinet, S. and Zaleski, S. Numerical simulation of droplets, bubbles, and waves: state of the art. Fluid Dynamic Resarch, 41, 2009.

[15] Fuster, D., Bagu, A., Boeck, T. Moyne, L. L., Leboisserier, A., Popinet, S., Ray, P., Scardovelli, R. and Zaleski, S. Simulation of primary atomization with octree adaptive mesh refinement and vof method. International Journal of Multiphase Flow, 35:550$565,2009$. 
[16] Gueyffier, D., Li, J., Nadim, A., Scardovelli, S. and Zaleski, S. Volume of fluid interface tracking with smoothed surface stress methods for three-dimensional flows. J. Comput. Phys., 152:423-456, 1999.

[17] Lafaurie, B., Nardone, C., Scardovelli, R., Zaleski, S. and Zanetti, G. Modelling merging and fragmentation in multiphase flows with surfer. J. Comput. Phys., 113:134-147, 1994.

[18] Popinet, S. An accurate adaptive solver for surface-tension-driven interfacial flows. $J$. Comput. Phys, 228:5838-5866, 2009.

[19] Popinet, S. and Zaleski, S. A front-tracking algorithm for accurate representation of surface tension. Int. J. Numer. Meth. Fluid, 30:775-793, 1999.

[20] Rider, W. J. and Kothe, B. D. Reconstructing volume tracking. J. Comput. Phys., 141:112-152, 1998.

[21] O.M.H. Rodriguez and R.V.A. Oliemans. Experimental study on oil-water flow in horizontal and slightly inclined pipes. International Journal of Multiphase Flow, 32:323343, 2006.

[22] Roma, A. M., Peskin C. S., and Berger M. J. An adaptive version of the immersed boundary method. J. Comput. Phys, 153:509-534, 1999.

[23] Rudman, M. Volume-tracking methods for interfacial flow calculations. Int. Jour. Numer. Methods Fluids, 24:671-691, 1997.

[24] Scardovelli, R. and Zaleski, S. Direct numerical simulation of free-surface and interfacial flow. Annu. Rev. Fluid Mech, 31:567-603, 1999.

[25] Scardovelli, R. and Zaleski, S. Analytical relations connecting linear interfaces and volume fractions in rectangular grids. J. Comput. Phys., 164:228-237, 2000.

[26] Seifollahi, M., Shirani, E. and Ashgriz, N. An improved method for calculation of interface pressure force in plic-vof methods. Chemical Engineering Journal, 27:1-23, 2008.

[27] Siti Aslina, H., Xu, X. Y. and Hewitt, G. F. Water local volume fraction on oil in water dispersion. J. Comput. Phys., 1:57-63, 2008.

[28] Tryggvason, G., Bunner, B., Esmaeeli, A., Juric, D., Al-Rawahi, N., Tauber, W., Han, J., Nas, S. and Jan, Y. J. A front-tracking method for the computations of multiphase flow. J. Comput. Phys., 169:708-759, 2001.

[29] Uhlmann, M. An immersed boundary method with direct forcing for the simulation of particulate flows. Journal of Computational Physics, 209:448-476, 2005.

[30] Hirt C. W. and B. D. Nichols. Volume of Fluid (VOF) method for the dynamics of free boundaries. J. Comput. Phys., 39:201-225, 1981.

[31] Wang, Z., Fan, J. and Luo, K. Combined multi-direct forcing and immersed boundary dor simulating flows with moving particles. International Journal of Multiphase Flow, 34:283-302, 2008. 